\title{
An in vitro analysis of the effect of acidosis on coagulation in chronic disease states - a thromboelastograph study
}

\author{
Authors: Hayden White, ${ }^{A}$ Robert Bird, ${ }^{B}$ Kellie Sosnowski ${ }^{C}$ and Mark Jones ${ }^{D}$
}

Thrombosis is a complication of many chronic illnesses. Chronic obstructive pulmonary disease (COPD) and diabetes mellitus are common medical conditions frequently associated with a hypercoagulable state. Acidaemia has been shown to reduce coagulation. COPD and diabetes mellitus during acute deterioration can present with a severe acidaemia. The impact of this acidaemia on coagulation is poorly studied. Patients presenting with a diagnosis of diabetic ketoacidosis or type II respiratory failure from COPD and a pH of less than 7.2 were included in our study. A coagulation screen and $a$ thromboelastograph (TEG) were performed on admission and 24 hours later. The mean $\mathrm{pH}$ on admission was 7.07 and mean base excess was $\mathbf{- 1 6 . 3}$. The activated partial thromboplastin time was associated with $\mathrm{pH}$ change but remained within the normal range (26-41 s). All other coagulation and TEG parameters failed to show evidence of association $(p>0.05)$. In the two models of non-haemorrhagic acidosis investigated, coagulation was not altered by the changes in $\mathrm{pH}$. More work is needed to understand the complex relationship between factors affecting coagulation in individual disease processes.

KEYWORDS: TEG, ROTEM, coagulation, acidosis, COPD, DKA

\section{Background}

Thrombosis is a concerning complication of many chronic illnesses. Chronic obstructive pulmonary disease (COPD) is frequently associated with a hypercoagulable state. ${ }^{1}$ Several studies have shown an increase in the incidence of both deep vein thrombosis (DVT) and pulmonary embolus (PE) in patients presenting with an acute exacerbation. A study by Riskallah and colleagues suggested the rate of PE to be as high as $24.7 \% .^{2}$ The reasons for this are numerous, but there is evidence that the increase in systemic inflammation associated with COPD, especially during acute exacerbations, results

Authors: A associate professor in critical care medicine, Griffiths University, Department of Intensive Care, Logan Hospital, Meadowbrook, Australia; ${ }^{B}$ director of hematology, Princess Alexandra Hospital, Woolloongabba, Australia; ${ }^{C}$ nurse unit manager, Logan Hospital, Meadowbrook, Australia; ${ }^{D}$ senior research fellow, University of Queensland, School of Population Health, Herston, Australia in a procoagulant state. ${ }^{3}$ As such, it would appear that many patients with COPD are at risk of DVT or PE.

Similarly, diabetes mellitus and diabetic ketoacidosis (DKA) are known to be associated with an increased risk of coagulation and atherosclerosis. ${ }^{4}$ Studies support the hypothesis that the metabolic insult of DKA alters the steady state of vascular endothelial cells, resulting in a prothrombotic state, which then predisposes to cerebrovascular complications. ${ }^{5}$ However, the severe acidosis associated with DKA could interfere with coagulation factors, rendering the patient hypocoagulable.

Acidosis has long been associated with hypocoagulopathy and poor outcomes. In fact, the deadly triad of hypothermia, acidosis and coagulopathy has long been associated with increased mortality after major trauma. ${ }^{6}$ Furthermore, data from in vitro studies in which acid (usually hydrochloric acid) is added to blood taken from healthy volunteers confirms that $\mathrm{pH}$ and coagulopathy are strongly correlated. However, the impact of acidosis in chronic disease populations is less clear.

The object of this study was to use the viscoelastic coagulation testing properties of the thromboelastograph (TEG) to characterise the impact of acidosis on coagulation during diabetic ketoacidosis or acute exacerbations of COPD with type II respiratory failure.

\section{Methods}

This single-site, prospective, clinical trial was done over a 12-month period to study the impact of $\mathrm{pH}$ on coagulation in patients presenting with DKA or COPD with respiratory acidosis using a TEG and other coagulation parameters. The study setting was a metropolitan intensive care unit (ICU). Ethics approval was granted by the Princess Alexandra Hospital Human Research Ethics Committee. The requirement for written informed consent was waived by the institutional review board.

All patients admitted to the Logan ICU (age $>18$ years) with $\mathrm{pH}$ less than 7.20 and either diabetic ketoacidosis or type II respiratory failure were eligible. Patients were excluded if on anticoagulants other than aspirin, or if they had severe sepsis, septic shock or shock for any other reason. Patients' fulfilling the inclusion criteria had blood samples collected and TEG assays using heparinase tubes performed on admission. All tests were repeated 24 hours later. Bloods for the TEG assay and arterial blood gases were taken and processed at the same time. Venous blood was collected by venipuncture from an antecubital vein. Venous samples were obtained simultaneously 
for thromboelastography, full blood count, coagulation profile, electrolyte and arterial blood gases $\left(\mathrm{pH}, \mathrm{CO}_{2}, \mathrm{HCO}_{3}\right.$, base excess, anion gap, lactate). The TEG sample was collected into a Vacuette 9NC coagulation tube containing 3.2\% buffered sodium citrate solution and allowed to completely fill the tube. It was gently inverted to ensure it was adequately mixed. Processing of the sample commenced within 30 minutes of venipuncture.

TEG assays were processed using the Haemoscope TEG 5000 thromboelastograph haemostasis analyser (Haemonetics Corporation). Routine machine quality control and standard calibrations were maintained. An electrical internal quality control (e-test) was done before each assay. Haemoscope TEG disposable cups (containing heparinase) and pins (Haemonetics Corporation) were placed in the analyser and pre-warmed to $37^{\circ} \mathrm{C}$. The process was initiated by adding $20 \mu \mathrm{L}$ of $0.2 \mathrm{M} \mathrm{CaCl}_{2}$ into the TEG test cup. $1 \mathrm{~mL}$ of the citrated blood sample was transferred to a vial of kaolin activator and mixed by gentle inversion. A $340 \mu \mathrm{L}$ aliquot of this blood sample was added to the cup and further mixed by raising and lowering the TEG pin three times before the analyser began to automatically process the sample.

Demographic data were collected, including participant age, sex and primary diagnosis. The body mass index was calculated. Coagulation profiles, biochemistry data and temperature were measured and recorded.

The TEG measures the viscoelastic properties of the clot as it forms and the technique has been previously described. ${ }^{7}$ The TEG haemostasis analyser provides thromboelastographic test results, including reaction time $(R)$, clot formation time $(K)$, alpha angle, maximum amplitude, time to maximum rate of thrombus generation (TG), maximum rate of thrombus generation (TMRTG), and total thrombus generation (TTG).

\section{Statistical analysis}

We based our sample size on an a priori power calculation in which 15 participants have $90 \%$ power to detect a 0.5 standard deviation change in TEG parameters, assuming a 5\% level of significance. We used random intercept linear mixed models to assess linear changes in measured variables over time and investigate potential associations between $\mathrm{pH}$ and other measured variables. An unstructured correlation was specified for all models.

\section{Results}

We included 18 patients in the study: five men and 13 women (mean age 38 years [range 17-81]; mean body mass index 26 [range 13-48]). Eleven patients presented with DKA and seven with type II respiratory failure. Descriptive statistics on the parameters by time (sample number) are described in Table 1.

Linear mixed models were used to assess linear change over time for all variables shown in Table 1 . The parameters that showed evidence of change are shown in Table 2. Positive estimates indicate that the parameter increased with time; the negative parameter for anion gap suggests that this variable decreased with time. Specifically, $\mathrm{pH}$ increased from 7.08 (SD 0.10) to 7.37 (SD 0.06; $\mathrm{p}<0.0001$ ), $\mathrm{HCO}_{3}$ increased from $13.89 \mathrm{mmol} / \mathrm{L}$ (SD 12.00) to $22.99 \mathrm{mmol} / \mathrm{L}(\mathrm{SD} 8.20 ; \mathrm{p}=0.0013)$

\begin{tabular}{|c|c|c|}
\hline & $\begin{array}{l}\text { Initial sample } \\
(n=18)\end{array}$ & $\begin{array}{l}\text { Sample at } 24 \\
\text { hours }(n=18)\end{array}$ \\
\hline$R(\min )$ & $6.86(2.43)$ & $6.41(2.23)$ \\
\hline$K(\min )$ & $1.87(0.93)$ & $1.98(0.95)$ \\
\hline$\alpha$ (degrees) & $64.32(8.83)$ & $62.49(9.43)$ \\
\hline $\mathrm{MA}(\mathrm{mm})$ & $62.01(9.53$ & $61.21(10.50)$ \\
\hline Coagulation index & $-0.53(3.46)$ & $-0.48(2.94)$ \\
\hline MRTG (mm/min) & $13.21(4.34)$ & $13.17(4.62)$ \\
\hline TMRTG (min) & $8.51(3.03)$ & $8.14(3.07)$ \\
\hline $\mathrm{TG}(\mathrm{mm} / \mathrm{min})$ & $748.50(111.64)$ & $740.66(123.80)$ \\
\hline $\mathrm{pH}$ & $7.08(0.10)$ & $7.37(0.06)$ \\
\hline $\mathrm{pCO}_{2}(\mathrm{~mm} \mathrm{Hg})$ & $49.06(46.81)$ & $41.06(17.07)$ \\
\hline $\mathrm{HCO}_{3}(\mathrm{mmol} / \mathrm{L})$ & $13.89(12.00)$ & $22.99(8.20)$ \\
\hline Base excess (mmol/L) & $-16.31(10.44)$ & $-2.44(7.09)$ \\
\hline Anion gap (mmol/L) & $13.67(6.80)$ & $5.59(2.98)$ \\
\hline Lactate (mmol/L) & $1.91(1.47)$ & $1.34(0.98)$ \\
\hline $\mathrm{pO}_{2}(\mathrm{~mm} \mathrm{Hg})$ & $90.83(49.41)$ & $82.72(25.92)$ \\
\hline APTT (s) & $28.52(5.84)$ & $31.18(7.24)$ \\
\hline Fibrinogen (mg/dL) & $3.62(1.20)$ & $3.47(1.24)$ \\
\hline PT (s) & $13.21(1.93)$ & $12.75(3.30)$ \\
\hline INR & $1.27(0.19)$ & $1.22(0.33)$ \\
\hline
\end{tabular}

Data are mean (SD). $\alpha=$ alpha angle; APTT = activated partial thromboplastin time; INR = international normalised ratio; $K=$ clot formation time; $\mathrm{MA}=$ maximum amplitude; $\mathrm{MRTG}=$ maximum rate of thrombus generation; $\mathrm{pCO}_{2}=$ partial pressure of $\mathrm{CO}_{2}$ in arterial blood; $\mathrm{pO}_{2}=$ partial pressure of oxygen; $\mathrm{PT}$ = prothrombin time; $R=$ reaction time; $\mathrm{TG}=$ total thrombus generation; TMRTG $=$ time to maximum rate of thrombus generation.

and activated partial thromboplastin time (APTT) from 28.52 $\mathrm{s}(\mathrm{SD} 5.84)$ to $31.18 \mathrm{~s}$ (SD 7.24; $\mathrm{p}=0.037)$, although this is still within the normal range of $26-41 \mathrm{~s}$.

In TEG measurements, $R$ time (representing the latency time before initial clot is formed) was reduced from 6.86 minutes (SD 2.3) to 6.41 minutes (SD2.23; $\mathrm{p}>0.05$ ), alpha angle (reflecting the rate of fibrin polymerisation and crosslinking) was decreased from $64.34^{\circ}$ (SD 8.83) to $62.49^{\circ}$ (SD 9.43; $\mathrm{p}>0.05$ ). Similarly, no significant changes of maximum amplitude, $K$ (both representing

\section{Table 2. Variables with evidence of linear change}

\section{over time.}

\begin{tabular}{llll} 
& Estimate & Standard error & p value \\
$\mathrm{pH}$ & 0.19 & 0.027 & $<0.0001$ \\
$\mathrm{HCO}_{3}(\mathrm{mmol} / \mathrm{L})$ & 6.13 & 1.65 & 0.0013 \\
Base excess $(\mathrm{mmol} / \mathrm{L})$ & 9.67 & 1.65 & $<0.0001$ \\
Anion gap $(\mathrm{mmol} / \mathrm{L})$ & -5.13 & 1.33 & 0.0010 \\
APTT $(\mathrm{s})$ & 1.64 & 0.73 & 0.037 \\
\hline
\end{tabular}

APTT $=$ activated partial thromboplastin time. 
R
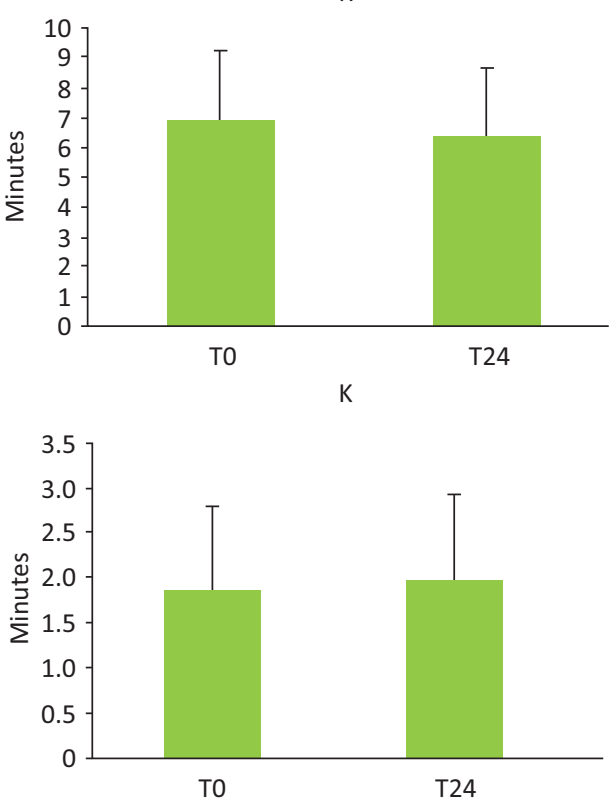

$\alpha$
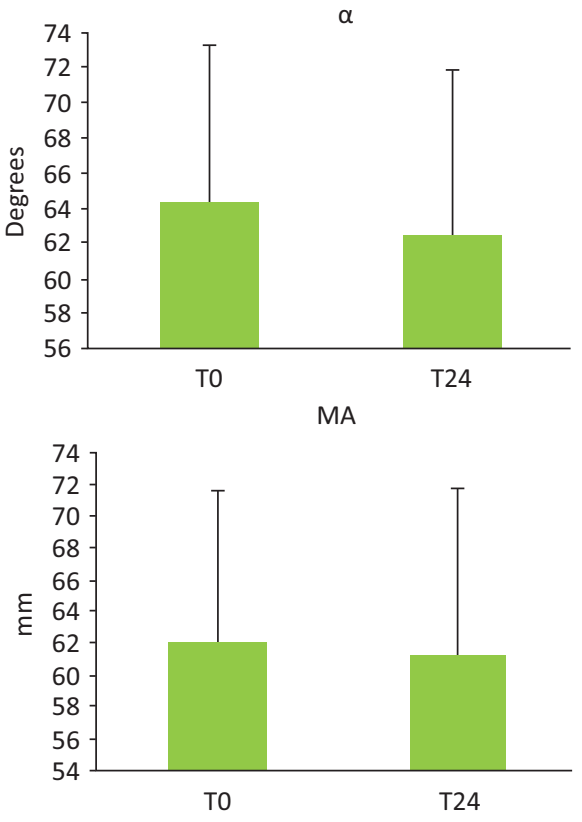

Fig 1. Thromboelastographic parameters from whole blood collected from patients presenting with diabetic ketoacidosis or type II respiratory failure. Data are mean and standard deviation. $\alpha=$ alpha angle (rate of clot formation); $K=$ clot formation time; $\mathrm{MA}=$ maximum amplitude; $R=$ reaction time; $\mathrm{T} 0=$ initial blood sample; $\mathrm{T} 24=$ 24 hours after initial sample.
In general, metabolic acidosis is associated with a hypocoagulant state. Following major trauma, hypothermia, acidosis and coagulopathy are associated with increased mortality. ${ }^{8}$ In vitro studies of blood removed from healthy volunteers and rendered acidaemic via infusion of hydrochloric acide confirm this. In a study by Engstrom and colleagues, hydrochloric acid was used to reduce blood $\mathrm{pH}$ to 6.83 . $^{6}$ Their rotational thromboelastometry studies demonstrated significant increase in clot formation time and reduction in alpha angle but not clotting time or maximum clot firmness. Another study by Meng and coworkers examined the effect of temperature and $\mathrm{pH}$ on factor VIIa in blood from healthy volunteers. ${ }^{9}$ They noted a marked reduction in the activity of factor VIIa-tissue factor complex and rate of prothrombin activation by factor Xa and Va complexes on phospholipid vesicles. Martini and colleagues infused hydrochloric acid into pigs while normothermic and hypothermic. ${ }^{10}$ They showed that, at a $\mathrm{pH}$ of 7.1, thrombin generation was decreased, APTT prolonged and the alpha angle decreased on TEG (suggesting delayed thrombin generation although still within the normal range); however, PT and other TEG measurements, such as maximum amplitude, $K$ and $R$ time, were normal. The fact that only some of the coagulation measurements were abnormal could reflect the varying effects of acidosis on different clotting factors. This finding was observed by Darlington and colleagues, who induced acidosis in pigs by either infusion of hydrochloric acid or via a haemorrage, hypoventilation model to a $\mathrm{pH}$ of 7.1. ${ }^{11}$ The acid group noted a decrease in platelets and fibrinogen (which has previously been demonstrated in hydrochloric acid infusion models) but also an increase in PT, APTT and activated clotting time and abnormal TEG functions ( $K$ time, alpha angle and maximum amplitude and prolonged thrombin generation). ${ }^{12}$

Our study demonstrates that the coagulopathy associated with acidosis is not a universal phenomenon. In fact, patients 
with COPD are prone to increased risk of coagulationassociated complications, especially during acute exacerbations. This finding has been confirmed in several studies. Gunen and colleagues found that $21 \%$ of COPD patients presenting with an acute exacerbation had demonstrable venous thromboembolism (VTE) or PE. ${ }^{13}$ D-dimers were noted to be significantly higher in the VTE group. Others have noted prevalence rates of VTE of $12.4 \%$ (95\% CI -2.2 to 27.1 ; $\mathrm{p}=$ 0.074 ) and rates of PE between $3.3 \%$ and $24.7 \% .^{2,14}$ One study showed a significant difference in DVT rates if the low molecular weight heparin nadroparin was administered (28\% in the placebo group versus $15 \%$ in the nadroparin group) ${ }^{15}$ Unfortunately, few studies report $\mathrm{pH}$ and when reported, $\mathrm{pH}$ tends to be within the normal range. To our knowledge, ours is the first study examining the effects of acidosis on the coagulation profile of patients with COPD presenting with an acute exacerbation and type II respiratory failure.

Similar results were noted in the patients with DKA. Diabetes mellitus has long been recognised as a procoagulant state. ${ }^{4}$ Various factors - including increased markers of coagulation activation with increased fibrin degradation products, tissue factor, and factors VII, XI, XII and decreased antithrombin and protein $\mathrm{C}$ - have been noted. The pathogenesis is complicated, with hyperglycemia leading to increases in cofactors in the acute setting while hyperglycosylation of proteins in the chronic setting leads to increased expression of tissue factor and mRNA. Carl and colleagues noted that protein C, free protein $\mathrm{S}$ and von Willebrand factor antigen were significantly influenced by DKA and its treatment. ${ }^{5}$ They postulated that the metabolic insult of DKA led to activation of endothelial cells. One of the postulates as to the cause of the procoagulant state is that DKA represents an inflammatory disease with increased C-reactive protein, cytokines and complement. ${ }^{16}$ This is reflected in abnormal endothelial function with raised von Willebrand Factor, tissue plasminogen activator and cellular adhesion molecules.

Children with DKA are at risk of DVT and cardiovascular diseases. ${ }^{17}$ In adults, increased homocysteine concentrations are found. An examination of 34 adult patients with uncomplicated DKA demonstrated evidence of endothelial injury, platelet activation, relative hypofibrinolysis and activation of the coagulation system, even in the absence of clinical signs of thrombosis. ${ }^{18}$ We were unable to demonstrate any clinically significant changes in coagulation during DKA. This is in keeping with a TEG study in children presenting with DKA, which failed to find any clinically significant changes in TEG parameters despite an average $\mathrm{pH}$ of $7.04 .^{19}$

The effects of acidosis on coagulation are complicated and poorly understood. Drops in $\mathrm{pH}$ to values below 7.2 reduce coagulation factor complex activities by as much as half and to $20 \%$ when $\mathrm{pH}$ reaches $6.8 .^{6}$ Animal studies have shown decreased factor VIIa-tissue factor complex and factor Xa-Va complex on phospholipid surfaces. ${ }^{10}$ Furthermore, both platelet and fibrinogen function are reduced. By contrast, COPD is associated with a procoagulant state. Mediators of coagulation can be found in the lung, including tissue factor. ${ }^{20}$ Patients display signs of enhanced activation of clotting factors in their airways, impaired function of the anticoagulant protein $\mathrm{C}$ system and attenuated fibrinolysis. Others have noted increased thrombin-antithrombin III complex, fibrinopeptide
A, $\beta$-thromboglobulin and tissue plasminogen activator concentrations. ${ }^{21}$ Wedzicha and colleagues found increased interleukin 6 and fibrinogen levels in keeping with increased inflammation. ${ }^{22}$ During exacerbations, there is evidence of an enhanced inflammation, with an increase in acute phase proteins, complement components, endothelial dysfunction, and variations of haemostasis mechanisms favouring coagulation. ${ }^{21,22}$ Endothelial dysfunction might be especially pronounced during acute exacerbations. Upregulation of adhesion molecules with subsequent increases in C-reactive protein, fibrinogen and complement activation products lead to atherogenesis.

On the basis of our results in the COPD and DKA populations, we have been unable to demonstrate the hypocoagulability associated with severe acidosis, or the hypercoagulability reportedly associated with acute exacerbations of COPD and severe DKA. Whether this is a result of the combined effects of the acidosis and underlying disease process is unclear. TEG provides an assessment of global coagulation and has demonstrated its efficacy in managing clotting abnormalities in a number of clinical settings. It has also proved useful as a marker of hypercoagulability associated with hepatic surgery, trauma, paediatric neurosurgery and disseminated intravascular coagulation. ${ }^{23-25}$ Although the humoral coagulation function can be readily assessed using TEG, the technique might be less able to offer insight into interactions between blood and endothelial cells, which could have a role in the hypercoagulability reported. This requires further study.

\section{Conclusion}

Although DKA and acute exacerbations of COPD are considered to be hypercoagulable states, we were unable to demonstrate any changes in the TEG parameters during an acute presentation. Conversely, the coagulopathy induced by severe acidosis does not appear to reduce the clotting capabilities of these disease states. This reflects the complex and often unpredictable nature of coagulation during periods when multiple disease processes are occurring simultaneously. More research is necessary to further examine the clinical impact and management of coagulation in these complex medical disorders.

\section{References}

1 Alessandri C, Basili S, Violi F et al. Hypercoagulability state in patients with chronic obstructive pulmonary disease. Chronic Obstructive Bronchitis and Haemostasis Group. Thromb Haemost 1994;72:343-6.

2 Rizkallah J, Man SFP, Sin DD. Prevalence of pulmonary embolism in acute exacerbations of COPD: a systematic review and metaanalysis. Chest 2009; 135:786-93.

3 Wouters EFM, Groenewegen KH, Dentener MA, Vernooy JHJ. Systemic inflammation in chronic obstructive pulmonary disease. Proc Am Thorac Soc 2007;4:626-34.

4 Carr ME. Diabetes mellitus: a hypercoagulable state. J Diabetes Complications 2001;15:44-54.

5 Carl GF, Hoffman WH, Passmore GG et al. Diabetic ketoacidosis promotes a prothrombotic state. Endocr Res 2003;29:73-82.

6 Engström M, Schött U, Romner B, Reinstrup P. Acidosis impairs the coagulation: a thromboelastographic study. J Trauma 2006;61:624-8.

7 Karon BS. Why is everyone so excited about thromboelastrography (TEG)? Clin Chim Acta Int J Clin Chem 2014;436C:143-8. 
8 Davenport R. Pathogenesis of acute traumatic coagulopathy: mechanisms of early posttraumatic coagulopathy. Transfusion 2013;53:23S-7S.

9 Meng ZH, Wolberg AS, Monroe DM, Hoffman M. The effect of temperature and $\mathrm{pH}$ on the activity of factor VIIa: implications for the efficacy of high-dose factor VIIa in hypothermic and acidotic patients. J Trauma Inj Infect Crit Care 2003;55:886-91.

10 Martini WZ, Pusateri AE, Uscilowicz JM, Delgado AV, Holcomb JB. Independent contributions of hypothermia and acidosis to coagulopathy in swine. J Trauma Inj Infect Crit Care 2005;58:1002-10.

11 Darlington DN, Kheirabadi BS, Delgado AV et al. Coagulation changes to systemic acidosis and bicarbonate correction in swine: J Trauma Inj Infect Crit Care 2011;71:1271-7.

12 Marumo M, Suehiro A, Kakishita E, Groschner K, Wakabayashi I. Extracellular $\mathrm{pH}$ affects platelet aggregation associated with modulation of store-operated $\mathrm{Ca}(2+)$ entry. Thromb Res 2001;104:353-60.

13 Gunen H, Gulbas G, In E, Yetkin O, Hacievliyagil SS. Venous thromboemboli and exacerbations of COPD. Eur Respir J 2010;35:1243-8.

14 Erelel M, Çuhadaro ǦÇ, Ece T, Arseven O. The frequency of deep venous thrombosis and pulmonary embolus in acute exacerbation of chronic obstructive pulmonary disease. Respir Med 2002;96:515-8.

15 Ambrosetti M, Ageno W, Spanevello A, Salerno M, Pedretti RFE. Prevalence and prevention of venous thromboembolism in patients with acute exacerbations of COPD. Thromb Res 2003;112:203-7.

16 Ileri NS, Büyükaşik Y, Karaahmetoğlu S et al. Evaluation of the haemostatic system during ketoacidotic deterioration of diabetes mellitus. Haemostasis 1999;29:318-25.

17 Bilici M, Tavil B, Dogru O, Davutoglu M, Bosnak M. Diabetic ketoasidosis is associated with prothrombotic tendency in children. Pediatr Hematol Oncol 2011;28:418-24.
18 Foster JR, Morrison G, Fraser DD. Diabetic ketoacidosis-associated stroke in children and youth. Stroke Res Treat 2011;2011:219706.

19 Tran TH, Al-Harfi I, Harle CC et al. Coagulation assessment in children with diabetic ketoacidosis. Pediatr Crit Care Med J Soc Crit Care Med World Fed Pediatr Intensive Crit Care Soc 2013;14:256-60.

20 De Boer JD, Majoor CJ, van't Veer C, Bel EHD, van der Poll T. Asthma and coagulation. Blood 2012;119:3236-44.

21 Ashitani J-I, Mukae H, Arimura Y, Matsukura S. Elevated plasma procoagulant and fibrinolytic markers in patients with chronic obstructive pulmonary disease. Intern Med Tokyo Jpn 2002;41:181-5.

22 Wedzicha JA, Seemungal TAR, MacCallum PK et al. Acute exacerbations of chronic obstructive pulmonary disease are accompanied by elevations of plasma fibrinogen and serum IL-6 levels. Thromb Haemost 2000;84:210-15.

23 Abdelfattah K, Cripps MW. Thromboelastography and rotational thromboelastometry use in trauma. Int J Surg 2015; published online 26 September. DOI:10.1016/j.ijsu.2015.09.036.

24 Krzanicki D, Sugavanam A, Mallett S. Intraoperative hypercoagulability during liver transplantation as demonstrated by thromboelastography. Liver Transpl 2013;19:852-61.

25 Panigada M, Zacchetti L, L'Acqua C et al. Assessment of fibrinolysis in sepsis patients with urokinase modified thromboelastography. PloS One 2015;10:e136463.

Address for correspondence: Dr H White, Department of Intensive Care, Logan Hospital, Queensland 4131, Australia. Email: hayden.white@health.qld.gov.au

'Smoking is the biggest avoidable cause of death and disability, and social inequality in health, in the UK.'

\section{Nicotine without smoke Tobacco harm reduction}

Since e-cigarettes became available in the UK in 2007, their use has been surrounded by medical and public controversy. This new 200-page report from the Royal College of Physicians examines the science, public policy, regulation and ethics surrounding e-cigarettes and other nontobacco sources of nicotine, and addresses these controversies and misunderstandings with conclusions based on the latest available evidence.

ISBN 978-1-86016-600-6 $£ 15$ including $p+p$ or free to download

Royal College of Physicians
Download the report: rcplondon.ac.uk/nicotine

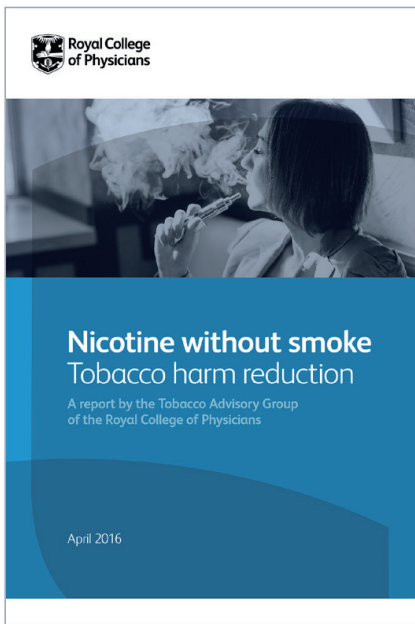

Order a copy: shop.rcplondon.ac.uk 